\title{
Transdisciplinary Application of Cross-Scale Resilience
}

\author{
Shana M. Sundstrom 1,*, David G. Angeler ${ }^{2}$, Ahjond S. Garmestani ${ }^{3}$, Jorge-H. García ${ }^{4}$ \\ and Craig R. Allen 5
}

1 Nebraska Cooperative Fish and Wildlife Unit, School of Natural Resources, University of Nebraska, Lincoln, NE 68583, USA

2 Department of Aquatic Sciences and Assessment, Swedish University of Agricultural Sciences, PO Box 7050, SE 75007 Uppsala, Sweden; E-Mail: david.angeler@slu.se

3 U.S. EPA, National Risk Management Research Laboratory, Cincinnati, OH 45268, USA;

E-Mail: garmestani.ahjond@epa.gov

4 CICERO, Center for International Climate and Environmental Research, Gaustadalleen 21, Oslo 0349, Norway; E-Mail: j.h.garcia@cicero.oslo.no

5 U.S. Geological Survey, Nebraska Cooperative Fish and Wildlife Unit, School of Natural Resources, University of Nebraska, Lincoln, NE 68583, USA; E-Mail: allencr@unl.edu

* Author to whom correspondence should be addressed; E-Mail: sundstrom.shana@gmail.com; Tel.: +1-360-773-8236.

External Editor: Marc A. Rosen

Received: 5 July 2014; in revised form: 2 September 2014 / Accepted: 17 September 2014 / Published: 2 October 2014

Abstract: The cross-scale resilience model was developed in ecology to explain the
emergence of resilience from the distribution of ecological functions within and across
scales, and as a tool to assess resilience. We propose that the model and the underlying
discontinuity hypothesis are relevant to other complex adaptive systems, and can be used to
identify and track changes in system parameters related to resilience. We explain the theory
behind the cross-scale resilience model, review the cases where it has been applied to
non-ecological systems, and discuss some examples of social-ecological, archaeological/
anthropological, and economic systems where a cross-scale resilience analysis could add a
quantitative dimension to our current understanding of system dynamics and resilience.
We argue that the scaling and diversity parameters suitable for a resilience analysis of
ecological systems are appropriate for a broad suite of systems where non-normative
quantitative assessments of resilience are desired. Our planet is currently characterized by 
fast environmental and social change, and the cross-scale resilience model has the potential to quantify resilience across many types of complex adaptive systems.

Keywords: complex adaptive systems; cross-scale dynamics; discontinuities; quantitative resilience; multidisciplinary application; social-ecological systems

\section{Introduction}

Scientists often have a poor understanding of the system-level behavior and dynamics of complex systems, such as ecosystems, economies, or integrated social-ecological-economic systems, whereas they are more likely to have a highly refined understanding of the components of complex systems, such as species or the behavior of individuals in an economy. The essence of a complex system, however, is that its behavior cannot be deduced from simply aggregating knowledge of the components. This fundamental constraint compels the need for tools that allow us to track the impact and consequences of localized changes or disturbances on system-level behavior and dynamics over time and space. The field of resilience science in ecology has studied resilience as an emergent system-level feature of complex ecological and social-ecological systems, and has developed a tool for quantitatively assessing ecosystem resilience, called the cross-scale resilience model. We argue that the cross-scale resilience model, which focuses on the emergence of resilience from the distribution of key elements within and across system scales [1] can be applied to other types of complex systems. The purpose of this paper is to explain the model and the theory underlying it, its relevance to other complex adaptive systems (CASs), and how it can be used in a resilience assessment.

Once the provenance of ecology [2], artificial life [3], and genetics [4,5], the application of complex adaptive systems theory to new fields has broadened considerably, from health care (data flows and human interactions) [6], food and water security [7], software development [8], business [9], legal systems [10], medical research [11], engineered systems such as electrical grids and traffic management [12,13], urban water systems, [14] and many more. Scientists are embracing a more complex view of system dynamics, and moving beyond long-held assumptions of linear equilibrium behavior for many different types of systems. Understanding universal, or at least broadly applicable, rules of complex systems behavior would assist the challenging task of understanding the "wicked problems" society faces, such as rapid environmental and social change including climate change, economic and socio-cultural challenges, biodiversity loss, and the degradation of social-ecological systems $[15,16]$.

Comparative analyses of complex systems have, in fact, demonstrated commonalities among distinctly different types of systems [17-21]. Both biological and non-biological complex systems appear to evolve and be structured by similar principles, leading to a limited set of possible topological structures, organization, dynamics and behavior that are to some extent universal across system types [21-24]. Levin (1998) proposed that the essential elements of a complex adaptive system can be reduced to three elements: "sustained diversity and individuality of components; localized interactions among the components; and an autonomous process, where based on the results of local interactions, a subset of the components is selected for replication or enhancement (p. 432)." From these essential elements flow the other key features of a CAS: adaptation and introduction of novelty [25], non-equilibrium 
dynamics as a result of the dispersed and local nature of selection, the absence of top-down global control, and the emergence of hierarchical organization and other emergent phenomena [26]. Of these features, scaling, hierarchical organization and the emergence of resilience is our focus. Resilience is the ability of a system to remain organized around the same set of processes, structures, and functions [27].

Within ecology, two parallel avenues of research have examined properties of CASs and their implications for system stability and resilience. The first, network theory, has uncovered rules of topological structure regarding the ways nodes are connected to each other using graph theory $[22,28]$, and examined the extent to which different topologies are resilient to random or targeted node loss [29-31]. In ecosystems, nodes are frequently modelled as species, connected to each other in food webs that generate emergent properties of information storage (such as genetic material), material and energy flow, resilience, and adaptive capacity [22,32-34]. Network theory has been widely applied to understand the effect of topological properties like connectance on the function and resilience of a broad array of CASs, from the internet, to social systems, and the brain [33,35,36]. However, network theory does not yet account for hierarchy and scaling in a non-arbitrary way when it considers scaling at all. Any scales identified are typically user-defined levels, as in when food-webs are stratified by trophic level [37].

The second research avenue, that of ecological resilience [27], was inspired by the multi-scalar and hierarchical organization of ecological systems. In particular, the discontinuity hypothesis was developed as a mechanistic explanation for the way species' interactions with the hierarchical, scaled nature of their environment structures communities [38]. The cross-scale resilience model extended the discontinuity hypothesis by providing a testable hypothesis for how system-level resilience can emerge from species' interactions with environmental structures and processes that vary with scale [1]. This model has provided one of the few quantitative measures of resilience available to date [39-41], despite the widespread uptake of the resilience concept. It specifically accounts for scaling and hierarchy in ways that network theory does not, but conversely, it can only speak to the relationships between objects in a system in a general way.

We propose that the cross-scale resilience model may describe fundamental patterns in CASs resulting from dynamics that are general to other types of hierarchical CASs. Here, we discuss some of the relevant theory underpinning ecological resilience, the discontinuity hypothesis, and the cross-scale resilience model, discuss recent examples from non-ecological systems, and then propose some systems for which we believe a cross-scale resilience analysis would be fruitful. We expect that a broader application of the cross-scale resilience model to different types of CASs will not only offer possibilities to increase our mechanistic understanding of the organization of ecological, social, and economic systems by complementing existing methods in those fields, but also help provide insight into management and policy challenges under fast-changing environmental and social baselines. Shared principles amongst systems has the pleasing consequence that theory, modeling and tools developed within one field for a particular type of CAS may be pertinent to another field, creating powerful opportunities for shared learning and collaboration. 


\subsection{Resilience}

The development of resilience theory has received considerable attention in recent years [24,40,42-45]. Ecological resilience is the ability of a system to remain organized around the same set of processes, structures, and functions [27]. The degree of resilience in a system is a measure of how much disturbance the system can buffer without moving into an alternative regime [1]. This is a distinctly different view of resilience than the more traditional engineering resilience, which defines resilience as the return time to equilibrium after a system has experienced a disturbance [46]. Engineering resilience presumes a single steady state, which is at odds with our current understanding of the dynamics of CASs. In practice, this means that once a CAS has shifted from Regime A to Regime B, an engineering view of resilience would incorrectly assume that the system would eventually rebound to Regime A without substantial intervention. Resilience theory has demonstrated that breaking the feedbacks that maintain the system in Regime B can be very difficult [47].

Resilience theory is built on an understanding of social-ecological systems as CASs, thus it assumes non-linear dynamics, and multiple possible basins of attraction governed by different regimes (i.e., different sets of processes). The ability to identify regime thresholds and provide early warnings of regime shifts is a vigorous area of current research $[42,48,49]$. Regime shifts are often abrupt, non-linear transitions between basins of attraction that occur when the threshold for a critical system driver is exceeded. When the resilience of a system is reduced, systems are more vulnerable to a potential regime shift. Fold-bifurcation threshold dynamics are common in ecological systems, where even a small change in conditions can trigger an abrupt regime shift if a bifurcation threshold is passed, and hysteresis, or the inability of a system to move backward and return to a previous regime, is possible [47,50]. Regime shifts in ecosystems epitomize the practical relevance of resilience research because the outcomes of regime shifts are uncertain, and frequently have negative consequences in the form of reduced ecosystem provisioning or increased human poverty $[51,52]$.

The relevance of resilience theory to other types of CASs is possible in part because order and pattern can emerge from the dynamics of self-organization in the absence of natural selection, merely from local interactions between agents [5]. Thus, although natural selection and evolution have corollaries in other fields - businesses as the objects of natural selection, or the evolution of CASs such as civilization, economies, or cities (see $[53,54]$ ), the emergence of higher-order phenomena such as resilience from lower-order localized interactions is not dependent on genetic-based natural selection [55]. It is increasingly clear that economies and other types of social systems have dynamics more appropriately described by the science of CASs than that of simple, linear dynamics, and tools like the discontinuity hypothesis and the cross-scale resilience model can be used to explore commonalities and differences in the basic dynamics of different types of CASs $[53,54]$.

\subsection{The Discontinuity Hypothesis}

The discontinuity hypothesis describes hierarchy and scaling in ecological systems as a result of structuring processes that occur over limited ranges of spatial and temporal scales. In ecological systems, some processes occur with high frequency and at small spatial scales, while others are slow and operate at large spatial extents, creating hierarchy and heterogeneity. Because the characteristic 
rate and extent of key structuring processes differ sufficiently, they create scale domains or ranges of scale over which patterns change monotonically or not at all. For example, Wiens [56] describes the scaling of transpiration, which is regulated by stomatal mechanisms at the scale of a leaf, but by climate at the scale of vegetation regions. Likewise, the processes that regulate the turnover of a pine needle differ fully from those that determine the location and extent of the boreal forest [38]. Scale domains are separated from each other by a non-linear transition (a discontinuity) to the next set of structuring processes $[38,56]$.

The discontinuity hypothesis is based on our understanding that species perceive and interact with their environment at scales that are relative to their body size, and persistence depends in part on how well a species' body mass allows it to take advantage of the resources available at a specific scale [38,57,58]. Animal body mass distributions for a given ecosystem consist of groups of similarly-sized species that exploit resources at similar scales. That is, each body mass group mirrors a specific scale of structure and resource availability in the ecosystem, such that the number of body mass groups indicates the number of scale domains present. These body mass groups are separated by gaps, which reflect a scale break (discontinuity), or transition to a new scale domain. Many animal communities have been tested for discontinuities with affirming results [38,59-62].

The discontinuity hypothesis relates to a general problem in ecology and other scientific disciplines regarding the quantification of scale in complex systems in non-arbitrary ways $[63,64]$. There have been few tools available for identifying the fundamental scales present in a system rather than defining levels of organization based on observer bias. Wiens [56] wrote, "we need non-arbitrary, operational ways of defining and detecting scales" and went on to ask, "How may we recognize domains of scale in a way that avoids arbitrary imposition of preconceived scales or hierarchical levels on natural variation?" The strength of the discontinuity analysis is that it is a tool for identifying the available scales of structure in a system without imposing human preconceptions. There are a variety of methods for detecting discontinuities, such as Bayesian classification and regression trees (BCART), Monte Carlo approaches (such as the Gap Rarity Index), and hierarchical cluster analysis [39,65-67]. These methods are used on rank-ordered body mass data for all the species in an ecological community (such as all the birds, mammals, or herpetofauna). Body mass can be obtained from general handbooks, as the patterns of aggregations and discontinuities in a system are highly robust to geographic variation and gender differences in body size [68]. Alternatively, discontinuities have been found by identifying where the fractal dimension of ecological structure changed abruptly, indicating that different structuring processes are dominant $[61,69]$. Time series modeling has also been used to identify temporal frequency patterns of groups of species at multiple scales within aquatic communities, as well as to evaluate aspects of cross-scale resilience, allowing for a more dynamic assessment of the discontinuity hypothesis [70,71]. All these tools are well established in the ecological literature and are readily applicable to other types of CASs. Once the pattern of aggregations and discontinuities has been identified using the methods described above (or other approaches), then the distribution of key elements thought to generate resilience can be evaluated, as per the cross-scale resilience model $[1,40,72,73]$. This approach identifies patterns in data, and the consistency of observed patterns with the posited cross-scale resilience model, but underlying causation is still debated [64,74]. 


\subsection{The Cross-Scale Resilience Model in Ecology}

The cross-scale resilience model emphasizes the compartmentalization by scale of the functional traits relevant for the maintenance of ecosystem processes. It posits that the distribution of functional traits within and across spatial and temporal scales in an ecological system is non-random, arises from processes of self-organization (positive interactions between structure, biota and process), and results in system-level resilience. Functional diversity, more so than species diversity, has proven crucial for the persistence and resilience of ecosystems and ecological functions such as primary productivity and pollination over time [75-77]. Species perform functions such as seed dispersal, pollination, decomposition, and nutrient cycling, and create feedbacks that maintain the ecosystem in a particular regime. The cross-scale resilience model posits that resilience derives from the overlapping but diverse functions within a particular scale domain, and the replication of function across the scales of a system [1]. Since disturbances do not affect all scales of a system equally, this pattern of functional distribution buffers the system against disrupted or lost functionality even if species are lost or reduced in abundance $[1,61,72,75,78]$.

Local interactions such as competition should drive species to differentiate in key ways to allow for co-existence [79]. Species that use similar resources are more likely to co-exist if they take advantage of different scales of resource distribution because this weakens their competitive interaction $[1,80]$. Species that interact with ecosystem structure at the same scales because of similar body sizes should tend to have a greater fitness if they utilize different resource types. A non-random distribution of species functions is thus a result of species interactions within a discontinuous template. Functional response diversity is also a component of resilience $[81,82]$. Response diversity is the degree to which species respond differently to a shared disturbance [83]. If all species belonging to the same functional group also respond similarly to environmental disturbance, then the response diversity is essentially one. If, however, species in the same functional group are differentially affected by a disturbance, then species less adversely affected can compensate for those species more severely impacted by the disturbance. The distribution of members of the same functional group across the scale domains of a system adds another layer of buffering against disturbances, because disturbances do not affect all scales of a system equally. The cross-scale resilience model proposes that resilience is enhanced when there is a diversity of functional groups within a scale domain, and a redundancy of functional groups across the scale domains, because this pattern will allow the system to absorb and buffer disturbances at a variety of scales due to compensatory dynamics [72,78]. Resilience is thus a consequence, and an emergent property of, complex discontinuous systems.

\subsection{An Example of the Cross-Scale Model in Non-Ecological Systems}

Only a small body of work has explicitly extended either the concept of discontinuous scaling in complex systems or the cross-scale model of resilience to non-ecological CASs [84-86]. Researchers applying the discontinuity analysis to city sizes found that the distribution was discontinuous, as city sizes within the south-eastern region of the United States fall into discrete size classes with growth rates that differ at different scales [84]. Discontinuities appear as gaps in rank-size distributions of city size within a region. Even though cities grew or shrank over time, the overall distribution pattern 
remained discontinuous, suggesting that the size classes reflect the scales of opportunity available in a given system and the processes that structure city size operate at discrete spatial and temporal scales [84]. In a follow-up to this work, Garcia et al. [87] analyzed the evolution of this city size distribution calculating Markov transition matrices that show the probability of a city moving up or down a size class or "state". They found that while short-term movements between size classes appear chaotic for the small to mid-sized cities, long-term transition probabilities across all size classes reveal relatively conservative system structure. Furthermore, the most persistent cities were the largest cities in the analysis, which lends further strength to the proposition that urban systems partition into levels in a dynamic hierarchy [88].

In another example, Garmestani et al. (2006) examined firm size distributions for manufacturing firms for the state of South Carolina, USA. They demonstrated that industrial sectors are comprised of firms that are clustered in size classes. They characterized resilience in industrial sectors [85] by following the cross-scale resilience model of Peterson et al. [1] and analyzed whether the coefficient of variation in employment trends (a proxy for resilience), was correlated to functional richness within an industrial sector. Functional richness was the number of size classes within an industrial sector, and the distribution of functional groups across the size classes, with functional groups represented by sub-sectors within an industrial sector. They expected that a more resilient industry would have more stable employment trends, and that this resilience would be correlated to having a higher functional diversity spread across more size classes within that industry. They found that manufacturing industries with greater functional richness spread across size classes exhibited less volatility in employment. The ability of small and large firms to adapt to variability in their "environment" without adding or shedding members suggests that economic resilience is enhanced when firms of different sizes emerge or are encouraged to emerge within industries.

\section{Applying the Cross-Scale Resilience Model to Other Complex Adaptive Systems}

There are four assumptions underlying the cross scale resilience model that are germane to other complex systems. We describe those assumptions and their implications using a well-established ecological example (Figure 1). The first assumption is that there are key processes in a complex system (A) that generate scale domains of structure (B) in a system. These scale domains are the "deep structure" of a system. Because the deep structure is scale specific and discontinuous, so too is the distribution of the components (C) (examples of $\mathrm{C}$ would be the organisms in ecological systems, or cities in regional systems) interacting with that structure. Resilience (D) emerges from the way critical functional attributes of these components are distributed across the discontinuous scale domains. Ideally, all these assumptions would be tested to understand CAS dynamics mechanistically, but assessing the processes that create the deep structure is often limited because some processes act over such broad spatial and temporal extents that they are challenging to measure. These limitations make an assessment of (A) difficult. However, determining (B) through (D) allows for the detection of patterns relevant for inferring resilience without the need to understand causal mechanisms. For example, Garmestani et al. $[85,89]$ found that both firm size and city size were reasonable analogues of animal body size, as firms and cities fell into distinct aggregations of similarly-sized firms with scale breaks between size classes, but they did not identify the scale-specific processes that generated the 
deep structure. Nevertheless, their identification of discontinuities and aggregations in system features such as firm size and city size provided insight into the dynamics of those urban and social systems, and their implications for resilience. This is useful for researchers applying tools across CAS types, because the distribution of key functional elements within and across the system scales should be a signature of emergent resilience (e.g., [90]).

Figure 1. Four layers of a cross-scale resilience model using simplified grassland. First, the key processes occur at discrete spatial and temporal scales, creating the heterogeneous and hierarchical scales of deep structure present in the system. Animals interact with the deep structure allometrically with their body size and are more likely to persist if their body mass is congruent with the scales of deep structure present. Resilience emerges from the non-random distribution of functions as performed by species within and across the scales of the system.

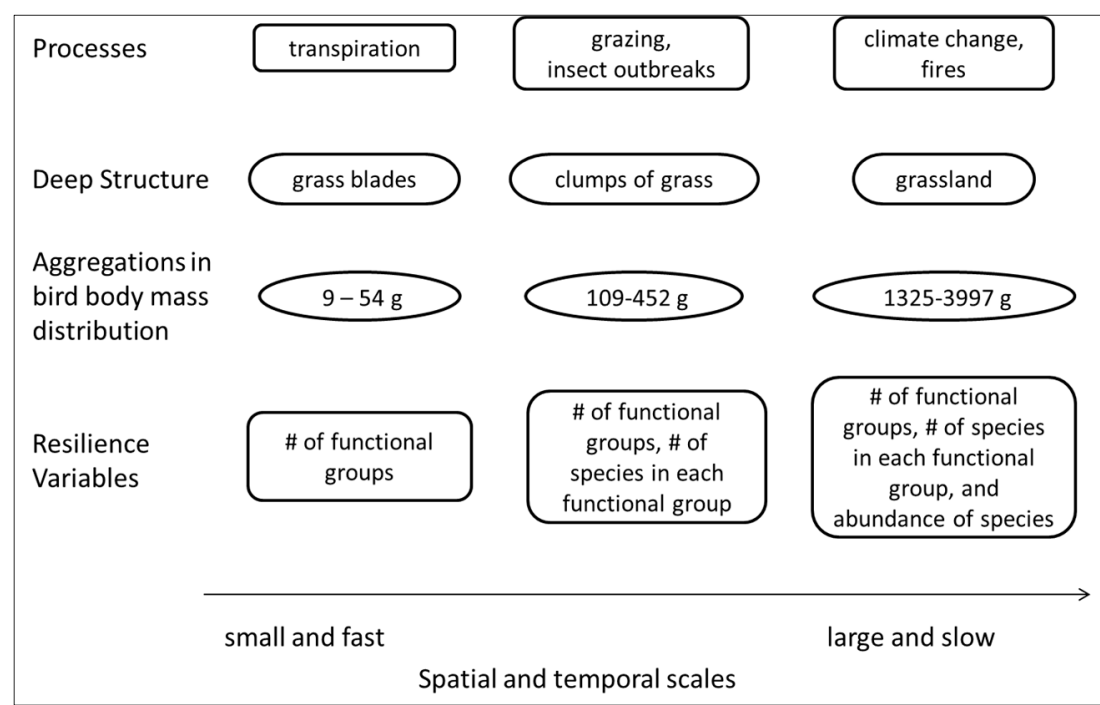

Because CASs are capable of existing in multiple different basins of attraction, defined by alternate regimes [48,49], it is important to understand the resilience attributes associated with each regime [91]. For instance, a lake can exist in a clear-water, oligotrophic regime, and a turbid-water, eutrophic regime. The turbid regime results from excessive nutrient loading and is undesirable because of reduced ecosystem service provisioning. The turbid state can also be resilient, making it extremely difficult to manage back into an oligotrophic regime [50]. Terrorist networks, composed of small cells that operate at discrete spatial and temporal scales and with limited cross-scale interactions, are also highly resilient to disruption because of the discontinuous structure of their organization. In both cases, this is not a resilience that is desirable [92]. Understanding what generates resilience and how to quantify it is of interest for any CAS upon which humans depend, because that knowledge can be applied to management efforts. Michael Batty expresses a similar sentiment on the limits of urban planning when he writes, "as we learn more about the functioning of such complex systems (cities), we will interfere less but in more appropriate ways" [93]. Other examples include social-ecological systems upon which we depend for food, water, recreation, and other values; our economic systems upon which much human well-being is dependent; socio-political systems which provide the stability to pursue a high-quality life; and the human body, including neurological and other biophysical human 
sub-systems. We describe the application of the cross-scale resilience model to several types of complex systems below (see also Table 1).

Table 1. Examples of types of complex adaptive systems and variables that may be conducive to a cross-scale resilience analysis.

\begin{tabular}{lll}
\hline \multicolumn{1}{c}{ Systems } & \multicolumn{1}{c}{ Variable } & \multicolumn{1}{c}{ Functional Attribute } \\
& & Emergency services \\
& & Production \\
& & Transportation options \\
Social-ecological/Urban & Population size & Employment diversification and evenness \\
Systems & & Energy grid \\
& & Food network \\
& & Types of open spaces \\
& & Ecosystem services \\
\hline \multirow{3}{*}{ Socio-cultural Systems } & Population size & Cultural diversity \\
& Government size/type & Educational opportunities (e.g., years of schooling) \\
& & Socio-economic diversity \\
& & Political upheaval \\
& GDP & Size of governed area \\
\hline \multirow{2}{*}{ Economic Systems } & Size classes of industry & Industry types (product diversity, export diversity), \\
& types within an economy & Natural Resource Dependence \\
& GINI coefficient & Employment (qualifications, redundancy) \\
& Stock market indexes & Standard-of-living measures \\
Systems & & Market sectors \\
\hline \multirow{2}{*}{ Socio-historical } & Population size & Access to environmental resources \\
& & Social connectivity within and across scales \\
& & Type of governance \\
\hline
\end{tabular}

\subsection{Social-Ecological}

The development of resilience theory in the last two decades has occurred almost exclusively within the realm of ecology. Much of the research conducted on ecosystems has treated humans as external to the system, but has developed quantitative methods to assess resilience (such as the cross-scale model) and the probability of regime shifts $[67,94]$, while another large body of work dealing explicitly with social-ecological systems has tended to focus on conceptual frameworks and assessments of resilience proxies [95-97]. Work focused on social-ecological resilience, could, in some instances, benefit from the quantitative identification of domains of scale, instead of relying on more arbitrary levels of organization within the system of interest. This allows for the specific identification of key processes structuring critical scales, or the distribution of functions or services deemed critical for maintaining social-ecological resilience. For example, Janssen et al. [98] provide a detailed case study assessment focused on configurations of social-ecological systems that have been resilient on century-time scales, but while they use scales to describe ecological/biological processes, they use "levels" to describe human organization structures. The implication is that scales in human systems are observer-dependent. We argue that while the key processes that constrain and structure human organizational structures are 
often different from those that structure ecological systems, they are nonetheless likely to be few and operate at discrete spatial and temporal scales that may or may not coincide with organizational levels.

Work on ecosystem services such as crop pollination [75] has shown that the stability of crop pollination is dependent on the response diversity and cross-scale distribution of the regional bee population, but the authors pre-selected seven scales of analysis, rather than using raw data to determine the scales at which bees interacted with the landscape [75]. Ecosystem services is a highly pertinent research topic given the rates of global land conversion, risks associated with climate change, and trends towards urbanization [16,99-101]. Objectively identifying the characteristic scales at which particular ecosystem services are distributed and the key processes or variables structuring those services would be of value. Furthermore, almost all ecological work focused on scales only considers spatial scales, by drawing buffers of arbitrary size around the focal phenomenon. This fails to identify the actual scales present, and does not account for a significant portion of the influence of scale - the temporal domain.

Urban systems can be considered a subset of social-ecological systems, as both cities and linked networks of cities are considered CASs [99]. Landscape ecologists have recently tackled regularities and deviations in patterns of development in urban systems, using metrics from hierarchical patch dynamics [102]. Using the cross-scale resilience model as an alternative method to identify key scales within urban systems would be an interesting validation of the landscape ecology approach. If similar scale domains were identified, the benefit of the cross-scale model is that it allows an evaluation of resilience by assessing how the distribution of key elements within and across those scales may impact urban resilience. Another body of work on urban systems has focused on uncovering universal urban scaling laws, usually in the form of power laws, that demonstrate how the size of a city scales with demographic, socio-economic and behavioral urban features such as crime rates, rate of innovation, and energy use $[103,104]$. It is likely that the power-law fit for many of these urban features masks deviations and discontinuities that reflect structuring processes that are not scale-invariant, and would allow researchers to determine why some cities are resilient and persistent over time, as compared with others [84,86]. Bettencourt et al. [103] write, "Scaling laws provide the average baseline behavior and the null-model for addressing how to rank cities meaningfully and assess the effects of local events, historical contingency and policy, independently of population size. We show how deviations from scaling laws can be used to construct truly local measures of a city's organization and dynamics". Ernstson et al. [99] argue that urban-ecological processes operate at multiple spatial-temporal scales and that cross-scale interactions are key to understanding system-level resilience. We suggest that deviations from scaling laws may not be confined to local contingency, but reflect structuring processes that are scale-specific and general across social-ecological systems, as in the work on human cognition that suggests that people can only meaningfully interact with roughly 150 other people, thus structuring social networks at that scale domain $[105,106]$. Just as the key processes that structure ecological systems are few and occur at characteristic spatio-temporal scales, the processes that structure human social organization appear to be as well [106-108].

An analysis of the rank-ordered distribution of the population of 179 U.S. Bureau of Economic Analysis-defined economic areas within the United States of America shows that there are six distinct size classes within the data set, which ranged from 80,415-23,285,781 people (Figure 2). An Economic Area reflects regional markets surrounding metropolitan or micropolitan statistical areas, 
which are defined based on commuting and newspaper readership data (see [109]) Size classes were identified using standard methods [66,86], and the number of classes was consistent with regional distributions analyzed by Garmestani et al. [89]. Time series analysis on this nation-wide BEA data set would allow researchers to examine robustness of size classes over time and the key processes generating the size classes, transitions of regions between size classes, and features of regions that promoted resilience and stability or were destabilizing over time.

Figure 2. Discontinuous distribution of 2011 Bureau of Economic Analysis (BEA)-defined economic regions for the United States of America. Bars represent size classes, while shading indicates what percentage of the 179 BEA regions fall into each size class. Bars are separated from adjacent size classes by significant gaps, or discontinuities.

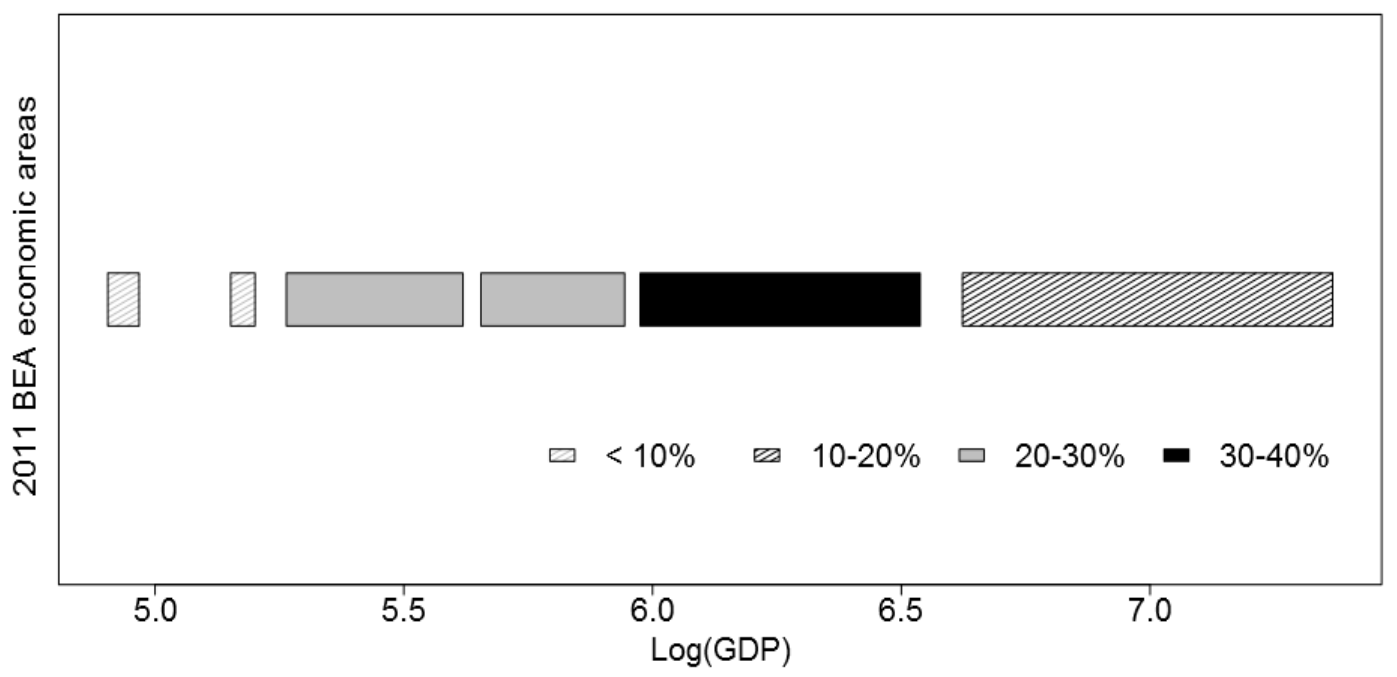

\subsection{Archaeology/Anthropology}

Human social organization has been explored by archaeologists, anthropologists and social historians from two primary viewpoints: the growth and development of human social organizations at all levels of organization over time, and the collapse of said organizations [54,110]. Despite the fact that human social organizations as CASs is widely accepted, few archaeologists have pursued complex systems theory as an avenue for exploring these dynamics of development and collapse over time (though see [105,111,112]). Interestingly, archaeology has recently embraced many of the basic concepts associated with resilience theory, especially that of the adaptive cycle [113-116]. The adaptive cycle provides a conceptual framework for understanding the dynamics of a system that moves through cycles of accumulation, collapse, and renewal, which were already a central focus in archaeological research. Much of this work, however, is largely descriptive and fails to objectively identify underlying scales of deep structure that might drive system dynamics over time [31]. Rather, it relies on human organizational levels and uses resilience theory and the adaptive cycle as an extended metaphor for explaining development and collapse dynamics in archaeological data [113,117]. Holling's discontinuity hypothesis was developed as a way to empirically test the adaptive cycle (Holling, personal communication), as it presumes hierarchal, nested, discrete scale domains, much as the cross-scale model was developed as a way to empirically test resilience within and across 
ecological communities. Part of the reason the adaptive cycle and resilience theory have not been embraced with more rigor might be a function of the inherently qualitative nature of the conceptswithout the cross-scale model as a means of testing whether key functional elements and the pattern of their distribution contributes to resilience, there is no recourse but description. One exception has been the work by Nelson et al. [118,119], which assessed whether diversity in household-level variables was correlated to resilience. The mixed results of those studies provide an important caveat to the use of the cross-scale model: first, the authors explored only diversity (and not redundancy) and its relationship to resilience, and at only two levels of subjectively-selected human organization (household and village). Second, all variables are not created equally. In other words, although animal body mass and the functions a species provides appear to incorporate many of the most critical elements of system structuring and system resilience, it is unknown what archaeological variables reflect the core processes and functions present in human social systems, and whether the archaeological material culture available to researchers, such as pottery styles, sufficiently represents the key scaling processes structuring human societies. How the basic concepts of diversity and redundancy within and across system scales translate into human material culture is an open question, but the cross-scale model would provide a quantitative platform with which to explore these not insignificant questions. Beekman and Baden [120] argue that rather than focusing on self-similarity across scales, a common feature of complex systems often embraced by researchers, the social sciences should concentrate on "phase transitions, emergence, and distinct scales of analysis with distinct rules".

Scaling in human population size has been treated by archaeology in a variety of ways and at various levels of organization. For the level of early villages, Rappaport [121] developed what he called the "Irritation Coefficient", which described the non-linear scaling relationship between an increase in population size and an increase in sources of irritation, or frequency of disputes. Gregory Johnson dubbed the phenomenon scalar stress, and argued from a social evolution perspective that due to the "Irritation Coefficient", expanding populations will either be forced to fission, and split into smaller and more manageable groups, or a higher-level governing layer capable of mitigating scalar stress will emerge [122]. Though archaeological evidence for fissioning in early villages and/or the emergence of a higher-level of institutional complexity is scarce due to the difficulties of data, scalar stress and its role in structuring scale domains of human population sizes remains a widely accepted theory $[121,123,124]$. The degree of acceptance despite the scarcity of hard evidence stems from work done by a wide array of theorists who have demonstrated that (a) the location of population size "hinge points", or thresholds, are common across human populations situated in very different environmental and cultural contexts; and (b) human cognitive factors such as short-term and long-term memory and limitations in information processing capabilities provide mechanisms for population hinge points $[105,107,108,125]$.

Subsequent work has shown that while the relationship between size and complexity is in general true, it can break down at narrow demographic ranges, as local context becomes more critical in structuring populations and their complexity [126] and this is congruent with the discontinuity hypothesis. A discontinuity analysis on archaeological data would be revealing of the key scale domains within which human populations fall, particularly as it uses raw data while previous work on archaeological data has used binned data, which can muddy the ability to find break points or clusters 
in rank-ordered data $[108,126]$. If human population sizes are structured by key processes operating at discrete spatial and temporal scales as Kosse [105] and others have suggested [107], then a resilience assessment could be conducted using the complexity variables already well-established in the archaeological literature [126]. Resilience could be synonymous, for example, with locational persistence at a comparable complexity, and its correlation with diversity and redundancy of environmental resources or social networks, or any other factors deemed critical for long-term persistence could be tested. If a discontinuity analysis detected similar aggregations of population sizes across disparate environments and cultures, this would indicate that the processes scaling populations are general to all humans, which would suggest that they are based on conservative patterns in primate evolution. One alternative is that some scales of aggregation are structured by primate evolution, while others may be more contingent on regional context, be it environmental or social, that nonetheless ought to be persistent and characteristic across types of environmental constraints or human political organization (e.g., collective leadership vs. autocratic leadership) [126]. Another alternative is that it may only be appropriate to apply discontinuity analysis and the cross-scale model to populations at regional scales, as is the case when applying these methods to ecological systems. If basic scaling processes can be associated with human population size classes, then comparative studies can begin assessing the degrees of resilience of various communities.

\subsection{Economic}

Since the Great Depression of the 1930s and the subsequent Keynesian Revolution, economics as a profession has been divided into two separate disciplines: microeconomics and macroeconomics. While the former studies micro fundamentals such as the specific market interactions of individuals and firms, the latter focuses on aggregates such as employment, interest rates, gross domestic product (GDP) and their fluctuations. Both the failure of macroeconomics to incorporate micro behavior, and conversely, the assumptions made when macro models do incorporate micro fundamentals has resulted in heated debates over the years (e.g., $[127,128]$ ). The micro-macro divide persists, to the point that most economists define themselves as one or the other. The inability of economics as a science to bridge the two distinct but interacting scales of behavior and dynamics underlines a need for alternative approaches.

Complex systems science has been slow to permeate economics, and despite recent progress [19,53,129-132], the study of economies as CASs has remained at the fringes of economics. Joseph Schumpeter was one of the few economists in the early 20th century who tried to understand the economy through a complex systems lens, but his ideas, emphasizing the dynamic nature of capitalist societies and business and economic cycles as endogenous behavior, have never been considered part of the mainstream [133]. Schelling [134] contributed to our understanding of selforganization in space, but less is known about temporal self-organization, such as the causes of the business cycles. Neoclassical economics, the predominant school of thought in economics for over a century, emphasizes economies as equilibrium systems with linear dynamics [129], which is an inappropriate characterization for economic systems over meaningful time scales $[19,53,135]$. That economies are examples of CASs has been convincingly argued [19,129,130]; we extend that characterization by arguing that socio-economic systems can be usefully analyzed from the perspective 
of discontinuous, hierarchical scales of structure, and the emergence of resilience from the distribution of key elements within and across the scales of a system.

Growth dynamics in economies appears to parallel those of ecosystems, suggesting that the evolutionary processes at work in both CASs are similar. Ecosystems and economies tend to increase in complexity over time, as they evolve increasingly complex structures to dissipate greater amounts of energy [20,53]. Stability or persistence over time occurs because of positive and negative feedback loops that reinforce processes of self-organization. A primary feature of stability in CASs comes from the trade-off between diversity and redundancy, rather than from the maximization or optimization of efficiency by maximizing diversity [136,137]. Increased diversity provides adaptive capacity because for evolution to occur systems must be able to change structurally in response to selective pressures [19], while redundancies provide a greater ability to withstand the loss of any one entity in the system [1,137]. Maximizing efficiency is destabilizing at the system level, as redundancies are critical in order to buffer disturbances. Lee et al. [138] found that though larger economies tended to be more diversified and thus have smaller relative fluctuations in growth, they were less diversified than would be expected if diversity increased linearly with size.

If size of economy is a key variable reflecting the scaling processes in economies, then the size distribution of economies should be discontinuous, reflecting the key scales of structuring processes. A cross-scale analysis of economies is predicted to confirm that the distribution of diversity within and across the scales of the system should be non-random, and those economies with increased diversity within scales and greater redundancies across scales ought to have greater resilience than less diversified economies. Guilmi et al. [139] found GDP per capita for countries between the 30th and 85th percentile fit a power law, suggesting that there are multiple scales of structuring processes in order to explain the tails of the distribution. Hidalgo and Haussman [140] examined the economic complexity of nations from a network perspective, moving away from traditional geographic or institutional explanations of economic growth. They focused on how the diversity of a country's labor inputs and the degree to which their exports are non-ubiquitous positively correlates with higher GDP, as well as being a good predictor of future GDP growth, demonstrating the importance of analyses that depart from a singular focus on system growth measures towards approaches that consider indicators of system resilience, and challenging classical theories on comparative advantage in economic development (e.g., [129]).

Ormerod [141] examined the resilience of capitalist economies to recessions, defining resilience as the duration of a recession, and found that capitalist economies were surprisingly resilient. Though this definition of resilience falls into the engineering category, presuming a single equilibrium state, it is one of the few to explicitly analyze how rapidly an economy is able to reorganize and rebound. He found that more than two-thirds of all recessions in the last 140 years lasted only a single year, regardless of the initial size of the recession. As there were a wide range of policy reactions to the 255 recessions, Ormerod [141] postulated that resilience to recessions is an inherent feature of economies, though without offering suggestions as to what structural features or mechanisms of a capitalist economy buffer the disturbance effects of a recession. The data did not fit a power-law, allowing us to reject the possibility that the probability of recessions is scale-invariant, and inviting the possibility that the cross-scale model could provide a method for probing the cross-scale characteristics contributing to economic resilience. 


\section{Tests of the Cross-Scale Model}

Applying the cross-scale model to non-ecological systems requires data that can be rank-ordered and is assumed to reflect key scaling processes, such as animal body masses in ecological systems, city sizes in a region, or firm sizes in an industrial sector (see Table 1 for examples). The data can be analyzed for discontinuities using one of several methods previously discussed (i.e., BCART, GRI, cluster analysis, fractal dimension, or time series analysis). The distribution of functionality within and across the scale domains identified in the discontinuity analysis is proposed to directly affect system-level resilience, so the data also needs to have a functional attribute associated with it. In ecological systems, this is represented by species' functional traits, while in economies it might be the diversification of sectors contributing to GDP, or in anthropological studies the diversity of food resources available to populations. The next step is to analyze the distribution of functional attributes within and across the scale domains identified $[41,72,78]$. Are the functions non-randomly distributed? Finally, the distribution pattern of function needs to be related to some measure of resilience, such as employment volatility [85], regime shifts in ecological systems [47], or socio-political upheavals [142]. We have outlined some systems that could be explored in this way, with examples of variables that can be rank-ordered, and functional attributes associated with those variables that can be analyzed for a measure of resilience (Table 1).

\section{Conclusions}

Biota, including humans, interact with the environment at distinct scales and create self-reinforcing patterns resistant to disturbance [143]. The multiple but distinct scales of self-organization and the distribution of function within and across scales generates system-level resilience [1]. Thus, a system's resilience is dependent upon the interactions between structure and dynamics at multiple scales.

Science has historically assessed complex systems in a reductionist fashion, decomposing the system into its constituent parts and attempting to understand and define the mechanisms driving each part. While the knowledge gained about the individual parts of the system has been invaluable, it has not led to the hoped-for insights into managing the system as a whole. Complex systems science has sought to address this by uncovering the general rules of behavior governing complex systems, rules that are not adducible from examining the constituents of the system. If resilience to disturbances is an emergent phenomenon of complex systems beyond ecosystems, then research into the key variables and patterns driving resilience provides an avenue for research tracking changes in resilience over time, conducting comparative analyses of resilience between systems, or as means of identifying critical variables on which policy and management actions should focus. The cross-scale resilience model provides a method for non-normative, quantitative assessments of resilience, and is, to our knowledge, one of the only methods available for doing so.

Our ability to identify and measure the key cross-scale variables that contribute to resilience provides society with more tools for prioritizing what system features and dynamics we try to manage. The increasing risk of concatenated crises suggests an urgency for doing so [144]. Just as network theory has contributed to our understanding of how network structure shapes a network's resilience to loss of nodes [145], the cross-scale resilience model contributes to our understanding of how the spatial 
and temporal distribution of key system variables buffers a system against disturbance and loss [1]. It is critical that we allocate our management resources towards system components and dynamics that underpin the fundamental resilience or behavior of the system, as opposed to identifying management targets based on subjective or reductionist views of what "matters" in the system.

In an economic example, managing product diversity through industrial policy demonstrates how an improved understanding of cross-scale dynamics could parlay into more influential management efforts (policies, in this case). Classical theories of comparative advantage suggest that product specialization maximizes social welfare at the country-level. However, we now know that product specialization alone makes economies highly vulnerable to external factors such as price fluctuations and price collapse in international markets. Cross-scale resilience suggests the implementation of economic policies whereby product diversity is at the core of economic development, as proposed in [146], but where system structure is recognized as multi-scaled and discontinuous and system dynamics are understood as cross-scale.

Although the cross-scale resilience model can be used to diagnose the relative resilience of a system, there are limitations to the approach. Assessing the distribution of functions within and across scales does not provide information regarding detailed relationships between and among functional elements, which is a strength of network approaches. The discontinuity hypothesis and the cross-scale resilience model allow for inferring general relationships, such as the supposition that interaction strengths between elements operating at the same scale will be stronger than those interacting across scales. The cross-scale resilience model, in its current form, also fails to evaluate the strength of feedbacks between biotic and abiotic variables, an important component of resilience. Similarly, although mechanisms have been inferred, the model does not directly provide information on the mechanisms underlying observed patterns. In its current form, the cross-scale resilience model also fails to account for abundance of elements and functions, or the role of rare elements in providing adaptive capacity [147] during disturbance and perturbation. Finally, it is not always possible to identify the key variables to include in an analysis, and the inclusion of the wrong types of variables may lead to spurious conclusions.

Utilization of the cross-scale resilience model may be most appropriate for comparative assessments, and as a relative measure. For example, the approach has been used to assess the resilience of subarctic lakes that are presumably threatened by global change, with vulnerability thought to increase with latitude [41]. However, Angeler et al. [41] found that boreal and circumboreal lakes were both surprisingly robust to changing environmental conditions, because the distribution of functional feeding guilds of invertebrates were similar within and across scales for all lakes. However, managing specific scales within the lakes was deemed impossible because the cross-scale distributions reflected environmental change at broad spatial extents with slow dynamics that are difficult to control. Nonetheless, the improved mechanistic insight into ecosystem dynamics would aid managers in focusing efforts on functional scales that are actually amenable to management action.

The application of these concepts to other types of systems is in its infancy, though work on the distribution of firm sizes and their "functional" role strongly suggests that similar processes are at work in structuring key patterns in economic systems. Many systems not discussed here would be conducive to a cross-scale resilience analysis, such as those found in neurology, immunology, physiology, microbiology (virology and bacteriology, as well as relationships with human health), 
paleo-ecology (e.g., diatoms), evolution, and political science. Many questions remain, but testing the cross-scale resilience model on these varied systems could lead to significant breakthroughs.

\section{Acknowledgments}

Shana M. Sundstrom was supported by an assistantship from the University of Nebraska-Lincoln School of Natural Resources, and by a Natural Sciences and Engineering Research Council of Canada doctoral grant. The views expressed in this paper are those of the authors and do not represent the views or policies of the U.S. Environmental Protection Agency. Support from the August T. Larsson foundation (NJ Faculty, Swedish University of Agricultural Sciences) is acknowledged. The Nebraska Cooperative Fish and Wildlife Research Unit is jointly supported by a cooperative agreement between the USGS, the Nebraska Game and Parks Commission, the University of Nebraska-Lincoln, the US Fish and Wildlife Service and the Wildlife Management Institute.

\section{Author Contributions}

Craig R. Allen, Ahjond S. Garmestani, and Shana M. Sundstrom conceived and outlined the manuscript, including the table. Shana M. Sundstrom wrote the manuscript, did the BEA analysis, and made the figures. David G. Angeler and Jorge-H. García contributed to sections. All provided substantive editorial contributions.

\section{Conflicts of Interest}

The authors declare no conflict of interest.

\section{References}

1. Peterson, G.D.; Allen, C.R.; Holling, C.S. Ecological resilience, biodiversity, and scale. Ecosystems 1998, 1, 6-18.

2. Lovelock, J.E. A numerical model for biodiversity. Philos. Trans. Biol. Sci. 1992, 338, 383-391.

3. Langton, C.G. Studying artificial life with cellular automata. Physica D 1986, 22, 120-149.

4. Serra, R.; Villani, M.; Graudenzi, A.; Kauffman, S.A. Why a simple model of genetic regulatory networks describes the distribution of avalanches in gene expression data. J. Theor. Biol. 2007, 246, 449-460.

5. Kauffman, S. At Home in the Universe; Oxford University Press: New York, NY, USA, 1995.

6. Tan, J.; Wen, H.J.; Awad, N. Health care and services delivery systems as complex adaptive systems. Commun. ACM 2005, 48, 36-44.

7. Villa, F.; Voigt, B.; Erickson, J.D. New perspectives in ecosystem services science as instruments to understand environmental securities. Philos. Trans. R. Soc. B 2014, doi:10.1098/rstb.2012.0286.

8. Batra, D.; VanderMeer, D.; Dutta, K. Extending agile principles to larger, dynamic software projects. J. Database Manag. 2011, 22, 73-92.

9. Mason, R.B. The external enviroment's effect on management and strategy: A complexity theory approach. Manag. Decis. 2007, 45, 10-28.

10. Ruhl, J.B. Managing systemic risk in legal systems. Indiana Law J. 2014, 89, 559-603. 
11. Greek, R.; Hansen, L.A. Questions regarding the predictive value of one evolved complex adaptive system for a second: Exemplified by the SOD1 mouse. Prog. Biophys. Mol. Biol. 2013, 113, 231-253.

12. Zhang, N.; Wang, F.-Y.; Zhu, F.; Zhao, D. DynaCAS : Computational experiments and decision support for ITS. IEEE Intell. Syst. 2008, 1541, 19-23.

13. Haghnevis, M.; Askin, R.G. A modeling framework for engineered complex adaptive systems. IEEE Syst. J. 2012, 6, 520-530.

14. Kanta, L.; Zechman, E. Complex adaptive systems framework to assess supply-side and demand-side management for urban water resources. J. Water Resour. Plan. Manag. 2014, 140, 75-85.

15. Vitousek, P.M.; Mooney, H.A.; Lubchenco, J.; Melillo, J.M. Human domination of earth's ecosystems. Science. 1997, 277, 494-499.

16. Millenium Ecosystem Assessment. Ecosystems and Human Well-Being: Biodiversity Synthesis; World Resources Institute: Washington, DC, USA, 2005.

17. Holling, C.S. Understanding the complexity of economic, ecological, and social systems. Ecosystems 2001, 4, 390-405.

18. Lansing, J.S. Complex adaptive systems. Annu. Rev. Anthropol. 2003, 32, 183-204.

19. Foster, J. From simplistic to complex systems in economics. Cambridge J. Econ. 2005, 29, 873-892.

20. Schneider, E.; Kay, J.K. Life as a manifestation of the second law of thermodynamics. Math. Comput. Model. 1994, 19, 25-48.

21. Bullmore, E.; Barnes, A.; Bassett, D.S.; Fornito, A.; Kitzbichler, M.; Meunier, D.; Suckling, J. Generic aspects of complexity in brain imaging data and other biological systems. Neuroimage 2009, 47, 1125-1134.

22. Barabási, A.-L. Scale-free networks: A decade and beyond. Science 2009, 325, 412-413.

23. Watts, D.J.; Strogatz, S.H. Collective dynamics of "small-world" networks. Nature 1998, 393, $440-442$.

24. Gunderson, L.H., Holling, C.S., Eds. Panarchy: Understanding Transformations in Human and Natural Systems; Island Press: Washington, DC, USA, 2002.

25. Levin, S.A. Ecosystems and the biosphere as complex adaptive systems. Ecosystems 1998, 1, 431-436.

26. Allen, C.R.; Holling, C.S. Novelty, adaptive capacity, and resilience. Ecol. Soc. 2010, 15, 24.

27. Holling, C.S. Resilience and stability of ecological systems. Annu. Rev. Ecol. Syst. 1973, 4, 1-23.

28. Cumming, G.S.; Bodin, Ö.; Ernstson, H.; Elmqvist, T. Network analysis in conservation biogeography: Challenges and opportunities. Divers. Distrib. 2010, 16, 414-425.

29. Dunne, J.A.; Williams, R.J. Cascading extinctions and community collapse in model food webs. Philos. Trans. R. Soc. Lond. B 2009, 364, 1711-1723.

30. Srinivasan, U.T.; Dunne, J.A.; Harte, J.; Martinez, N.D. Response of complex food webs to realistic extinction sequences. Ecology 2007, 88, 671-682.

31. Uden, D.R.; Hellman, M.L.; Angeler, D.G.; Allen, C.R. The role of reserves and anthropogenic habitats for functional connectivity and resilience of ephemeral habitats. Ecol. Appl. 2014, in press. 
32. Montoya, J.M.; Solé, R.V. Topological properties of food webs: From real data to community assembly models. Oikos 2003, 102, 614-622.

33. Pascual, M., Dunne, J.A., Eds. Ecological Networks: Linking Structure to Dynamics in Food Webs; Oxford University Press: Oxford, UK, 2005.

34. Jørgensen, S.E.; Fath, B.D. Application of thermodynamic principles in ecology. Ecol. Complex. 2004, 1, 267-280.

35. Barabási, A.-L. Network medicine-From obesity to the "diseasome". N. Engl. J. Med. 2007, 357, 404-407.

36. Barabási, A.-L. Linked; Plume: New York, NY, USA, 2003; pp. 1-304.

37. Bascompte, J.; Melian, C.J.; Sala, E. Interaction strength combinations and the overfishing of a marine food web. Proc. Natl. Acad. Sci. 2005, 102, 5443-5447.

38. Holling, C.S. Cross-scale morphology, geometry, and dynamics of ecosystems. Ecol. Monogr. 1992, 62, 447-502.

39. Stow, C.; Allen, C.R.; Garmestani, A.S. Evaluating discontinuities in complex systems: Toward quantitative measures of resilience. Ecol. Soc. 2007, 12, 26.

40. Allen, C.R.; Gunderson, L.H.; Johnson, A.R. The use of discontinuities and functional groups to assess relative resilience in complex systems. Ecosystems 2005, 8, 958-966.

41. Angeler, D.G.; Allen, C.R.; Johnson, R.K. Measuring the relative resilience of subarctic lakes to global change: redundancies of functions within and across temporal scales. J. Appl. Ecol. 2013, 50, 572-584.

42. Folke, C.S.; Carpenter, S.R.; Walker, B.H.; Scheffer, M.; Elmqvist, T.; Gunderson, L.H.; Holling, C.S. Regime shifts, resilience, and biodiversity in ecosystem management. Annu. Rev. Ecol. Evol. Syst. 2004, 35, 557-581.

43. Walker, B.H.; Salt, D. Resilience Practice: Building Capacity to Absorb Disturbance and Maintain Function; Island Press: Washington, DC, USA, 2012; pp. 1-248.

44. Carpenter, S.R.; Brock, W.A. Spatial complexity, resilience, and policy diversity: Fishing on lake-rich landscapes. Ecol. Soc. 2004, 9, 1-31.

45. Cumming, G.S. Spatial Resilience in Social-Ecological Systems; Springer Science: New York, NY, USA, 2011; pp. 1-308.

46. Holling, C.S.; Meffe, G.K. Command and control and the pathology of natural resource managment. Conserv. Biol. 1996, 10, 328-337.

47. Scheffer, M.; Carpenter, S.R.; Foley, J.A.; Folke, C.; Walker, B. Catastrophic shifts in ecosystems. Nature 2001, 413, 591-596.

48. Scheffer, M.; Carpenter, S.R.; Lenton, T.M.; Bascompte, J.; Brock, W.A.; Dakos, V.; van de Koppel, J.; van de Leemput, I.A.; Levin, S.A.; van Nes, E.H.; et al. Anticipating critical transitions. Science. 2012, 338, 344-348.

49. Biggs, R.; Carpenter, S.R.; Brock, W.A. Turning back from the brink: detecting an impending regime shift in time to avert it. Proc. Natl. Acad. Sci. 2009, 106, 826-831.

50. Scheffer, M.; Carpenter, S.R. Catastrophic regime shifts in ecosystems: Linking theory to observation. Trends Ecol. Evol. 2003, 18, 648-656.

51. Moberg, F.; Folke, C.S. Ecological goods and services of coral reef ecosystems. Ecol. Econ. 1999, 29, 215-233. 
52. Crépin, A.-S.; Biggs, R.; Polasky, S.; Troell, M.; de Zeeuw, A. Regime shifts and management. Ecol. Econ. 2012, 84, 15-22.

53. Beinhocker, E.D. The Origin of Wealth: Evolution, Complexity, and the Radical Remaking of Economics; Harvard Business School Press: Boston, MA, USA, 2006; pp. 1-526.

54. Tainter, J.A. The Collapse of Complex Societies; Cambridge University Press: Cambridge, UK, 1988; pp. 1-262.

55. Van den Bergh, J.C.J.M. Evolutionary thinking in environmental economics. J. Evol. Econ. 2007, 17, 521-549.

56. Wiens, J.A. Spatial scaling in ecology. Funct. Ecol. 1989, 3, 385-397.

57. Peters, R. The Ecological Implications of Body Size; Cambridge University Press: Cambridge, UK, 1983.

58. Fisher, J.T.; Anholt, B.; Volpe, J.P. Body mass explains characteristic scales of habitat selection in terrestrial mammals. Ecol. Evol. 2011, 1, 517-528.

59. Allen, C.R.; Garmestani, A.S.; Havlicek, T.D.; Marquet, P.A.; Peterson, G.D.; Restrepo, C.; Stow, C.; Weeks, B.E. Patterns in body mass distributions: Sifting among alternative hypotheses. Ecol. Lett. 2006, 9, 630-643.

60. Havlicek, T.; Carpenter, S.R. Pelagic species size distributions in lakes: Are they discontinuous? Limnol. Oceanogr. 2001, 46, 1021-1033.

61. Nash, K.; Graham, N.; Wilson, S.; Bellwood, D.R. Cross-scale habitat structure drives fish body size distributions on coral reefs. Ecosystems 2013, 16, 478-490.

62. Lambert, W.D. Functional convergence of ecosystems: Evidence from body mass distributions of North American Late Miocene mammal faunas. Ecosystems 2006, 9, 97-118.

63. Gibson, C.C.; Ostrom, E.; Ahn, T.K. The concept of scale and the human dimensions of global change: A survey. Ecol. Econ. 2000, 32, 217-239.

64. Nash, K.; Allen, C.R.; Angeler, D.G.; Barichievy, C.; Eason, T.; Garmestani, A.S.; Graham, N.; Granholm, D.; Knutson, M.G.; Nelson, R.J.; et al. Discontinuities, cross-scale patterns and the organization of ecosystems. Ecology 2014, 95, 654-667.

65. Allen, C.R.; Holling, C.S. Cross-scale morphology. Encycl. Environmetrics 2002, 1, 450-452.

66. Allen, C.R.; Holling, C.S. Cross-scale structure and scale breaks in ecosystems and other complex systems. Ecosystems 2002, 5, 315-318.

67. Allen, C.R., Holling, C.S., Eds. Discontinuities in Ecosystems and Other Complex Systems; Island Press: New York, NY, USA, 2008.

68. Sundstrom, S.M. The Textural Discontinuity Hypothesis and Its Relationship to Biodiversity, Extinction Risk, and Ecosystem Resilience. Master's Thesis, University of Calgary, Calgary, AB, Canada, 2009.

69. Li, B.-L. Fractal geometry applications in description and analysis of patch patterns and patch dynamics. Ecol. Model. 2000, 132, 33-50.

70. Angeler, D.G.; Drakare, S.; Johnson, R.K. Revealing the organization of complex adaptive systems through multivariate time series modeling. Ecol. Soc. 2011, 16, 5.

71. Angeler, D.G.; Trigal, C.; Drakare, S.; Johnson, R.K.; Goedkoop, W. Identifying resilience mechanisms to recurrent ecosystem perturbations. Oecologia 2010, 164, 231-241. 
72. Sundstrom, S.M.; Allen, C.R.; Barichievy, C. Species, functional groups, and thresholds in ecological resilience. Conserv. Biol. 2012, 26, 305-314.

73. Angeler, D.G.; Johnson, R.K. Algal invasions, blooms and biodiversity in lakes: Accounting for habitat-specific responses. Harmful Algae 2013, 23, 60-69.

74. Vergnon, R.; van Nes, E.H.; Scheffer, M. Emergent neutrality leads to multimodal species abundance distributions. Nat. Commun. 2012, 3, 1-6.

75. Winfree, R.; Kremen, C. Are ecosystem services stabilized by differences among species? A test using crop pollination. Proc. R. Soc. B 2009, 276, 229-237.

76. Chillo, V.; Anand, M.; Ojeda, R.A. Assessing the use of functional diversity as a measure of ecological resilience in arid rangelands. Ecosystems 2011, 14, 1168-1177.

77. Schmitz, O.J. Resolving Ecosystem Complexity; Princeton University Press: Princeton, NJ, USA, 2010; pp. 1-176.

78. Wardwell, D.A.; Allen, C.R.; Peterson, G.D.; Tyre, A.J. A test of the cross-scale resilience model: Functional richness in Mediterranean-climate ecosystems. Ecol. Complex. 2008, 5, 165-182.

79. Holt, R.D. Bringing the Hutchinsonian niche into the 21st century: Ecological and evolutionary perspectives. Proc. Natl. Acad. Sci. 2009, 106, 19659-19665.

80. Ritchie, M.E. Scale, Heterogeneity, and the Structure and Diversity of Ecological Communities; Princeton University Press: Princeton, NJ, USA, 2009; pp. 1-232.

81. Elmqvist, T.; Folke, C.S.; Nystrom, M.; Peterson, G.D.; Bengtsson, J.; Walker, B.H.; Norberg, J. Response diversity, ecosystem change, and resilience. Front. Ecol. Environ. 2003, 1, 488-494.

82. Laliberte, E.; Wells, J.A.; DeClerck, F.; Metcalfe, D.J.; Catterall, C.P. Land-use intensification reduces functional redundancy and response diversity in plant communities. Ecol. Lett. 2010, 13, 76-86.

83. Bellwood, D.R.; Hughes, T.P.; Folke, C.S.; Nystrom, M. Confronting the coral reef crisis. Nature 2004, 429, 827-833.

84. Garmestani, A.S.; Allen, C.R.; Gallagher, C.M.; Mittelstaedt, J.D. Departures from Gibrat's Law, discontinuities and city size distributions. Urban Stud. 2007, 44, 1997-2007.

85. Garmestani, A.S.; Allen, C.R.; Mittelstaedt, J.D.; Stow, C.A.; Ward, W.A. Firm size diversity, functional richness, and resilience. Environ. Dev. Econ. 2006, 11, 533.

86. Garmestani, A.S.; Allen, C.R.; Gallagher, C.M. Power laws, discontinuities and regional city size distributions. J. Econ. Behav. Organ. 2008, 68, 209-216.

87. García, J.H.; Garmestani, A.S.; Karunanithi, A.T. Threshold transitions in a regional urban system. J. Econ. Behav. Organ. 2011, 78, 152-159.

88. Eason, T.; Garmestani, A.S. Cross-scale dynamics of a regional urban system through time. Reg. Dev. 2012, 36, 55-76.

89. Garmestani, A.; Allen, C.; Bessey, K.M. Time-series analysis of clusters in city size distributions. Urban Stud. 2005, 42, 1507-1515.

90. Angeler, D.G.; Allen, C.R.; Johnson, R.K. Insight on invasions and resilience derived from spatiotemporal discontinuities of biomass at local and regional scales. Ecol. Soc. 2012, 17, 32.

91. Angeler, D.G.; Allen, C.R.; Rojo, C.; Alvarez-Cobelas, M.; Rodrigo, M.A.; Sánchez-Carrillo, S. Inferring the relative resilience of alternative states. PLoS One 2013, 8, e77338. 
92. Bousquet, A. Complexity theory and the War on Terror: Understanding the self-organizing dynamics of leaderless jihad. J. Int. Relations Dev. 2012, 15, 345-369.

93. Batty, M. The size, scale, and shape of cities. Science. 2008, 319, 769-771.

94. Scheffer, M.; Bascompte, J.; Brock, W.A.; Brovkin, V.; Carpenter, S.R.; Dakos, V.; Held, H.; van Nes, E.H.; Rietkerk, M.; Sugihara, G. Early-warning signals for critical transitions. Nature 2009, 461, 53-59.

95. Walker, B.H.; Carpenter, S.R.; Anderies, J.M.; Abel, N.; Cumming, G.S.; Janssen, M.; Lebel, L.; Norberg, J.; Peterson, G.D.; Prichard, R. Resilience management in social-ecological systems: A working hypothesis for a participatory approach. Conserv. Ecol. 2002, 6, 14.

96. Cumming, G.S.; Barnes, G.; Perz, S.; Schmink, M.; Sieving, K.E.; Southworth, J.; Binford, M.; Holt, R.D.; Stickler, C.; Holt, T. An exploratory framework for the empirical measurement of resilience. Ecosystems 2005, 8, 975-987.

97. Adger, W.N.; Brown, K.; Nelson, D.R.; Berkes, F.; Eakin, H.; Folke, C.S.; Galvin, K.; Gunderson, L.H.; Goulden, M.; O’Brien, K.; et al. Resilience implications of policy responses to climate change. Clim. Chang. 2011, 2, 757-766.

98. Janssen, M.A.; Anderies, J.M.; Ostrom, E. Robustness of social-ecological systems to spatial and temporal variability. Soc. Nat. Resour. 2007, 20, 307-322.

99. Ernstson, H.; Leeuw, S.E.; Redman, C.L.; Meffert, D.J.; Davis, G.; Alfsen, C.; Elmqvist, T. Urban transitions: On urban resilience and human-dominated ecosystems. Ambio 2010, 39, 531-545.

100. Rockström, J.; Steffen, W.; Noone, K.; Persson, A.; Chapin, F.S. A safe operating space for humanity. Nature 2009, 461, 472-475.

101. Costanza, R.; d’Arge, R.; de Groot, R.; Farber, S.; Grasso, M.; Hannon, B.; Limburg, K.; Naeem, S.; O'Neill, R.V.; Paruelo, J.; et al. The value of the world's ecosystem services and natural capital. Nature 1997, 387, 253-260.

102. Wu, J.; Jenerette, G.D.; Buyantuyev, A.; Redman, C.L. Quantifying spatiotemporal patterns of urbanization: The case of the two fastest growing metropolitan regions in the United States. Ecol. Complex. 2011, 8, 1-8.

103. Bettencourt, L.M.A.; Lobo, J.; Strumsky, D.; West, G.B. Urban scaling and its deviations: Revealing the structure of wealth, innovation and crime across cities. PLoS One 2010, 5, e13541.

104. Bettencourt, L.M.A.; Lobo, J.; Helbing, D.; Kühnert, C.; West, G.B. Growth, innovation, scaling, and the pace of life in cities. Proc. Natl. Acad. Sci. 2007, 104, 7301-7306.

105. Kosse, K. Some regularities in human group formation and the evolution of societal complexity. Complexity 2001, 6, 60-64.

106. Aiello, L.A.; Dunbar, R.I.M. Neocortex size, group size, and the evolution of language. Curr. Anthropol. 1993, 34, 184-193.

107. Dunbar, R.I.M. Cognitive constraints on the structure and dynamics of social networks. Gr. Dyn. Theory Res. Pract. 2008, 12, 7-16.

108. Kosse, K. Group-size and societal complexity: Thresholds in the long-term memory. J. Anthropol. Archaeol. 1990, 9, 275-303.

109. Johnson, K.P.; Kort, J.R. 2004 Redefinition of the BEA Economic Areas. Available online: http://www.bea.gov/scb/pdf/2004/11November/1104Econ-Areas.pdf (accessed on 17 September 2014). 
110. Diamond, J. Collapse: How Societies Choose to Fail or Succeed; Viking: New York, NY, USA, 2004.

111. Yoffee, N. Memorandum to: Murray Gell- Mann Concerning: The Complications of Complexity in the Prehistoric Southwest. Available online: http://www.santafe.edu/media/workingpapers/ 93-09-057.pdf (accessed on 17 September 2014).

112. Coombes, P.; Barber, K. Environmental determinism in Holocene research: Causality or coincidence? Area 2005, 37, 303-311.

113. Dunning, N.P.; Beach, T.P.; Luzzadder-Beach, S. Kax and kol: Collapse and resilience in lowland Maya civilization. Proc. Natl. Acad. Sci. 2012, 109, 3652-3657.

114. Hegmon, M.; Peeples, M.A.; Kinzig, A.P.; Kulow, S.; Meegan, C.M.; Nelson, M.C. Social transformation and its human costs in the Prehispanic U.S. Southwest. Am. Anthropol. 2008, 110, 313-324.

115. Nelson, D.R.; Adger, W.N.; Brown, K. Adaptation to environmental change: Contributions of a resilience framework. Annu. Rev. Environ. Resour. 2007, 32, 395-419.

116. Redman, C.L. Resilience theory in archaeology. Am. Anthropol. 2005, 107, 70-77.

117. Ekblom, A. Livelihood security, vulnerability and resilience: A historical analysis of Chibuene, southern Mozambique. Ambio 2012, 41, 479-489.

118. Nelson, C.; Hegmon, M.; Kulow, S.; Schollmeyer, K.G. Archaeological and ecological perspectives on reorganization: A case study from the Mimbres region of the U.S. Southwest. Am. Antiq. 2006, 71, 403-432.

119. Nelson, M.C.; Hegmon, M.; Kulow, S.R.; Peeples, M.A.; Kintigh, K.W.; Kinzig, A.P. Resisting diversity: A long-term archaeological study. Ecol. Soc. 2011, 16, 25.

120. Beekman, C.S.; Baden, W.W. Continuing the Revolution. In Nonlinear Models for Archaeology and Anthropology; Beekman, C.S., Baden, W.W., Eds.; Ashgate Publishing: Hampshire, UK, 2005; pp. 1-12.

121. Bandy, M.S. Fissioning, scalar stress, and social evolution in early village societies. Am. Anthropol. 2004, 106, 322-333.

122. Johnson, G. Organizational Structure and Scalar Stress. In Theory and Explanation in Archaeology; Renfrew, C., Rowlands, M., Segraves, B.A., Eds.; Academic Press: New York, NY, USA, 1982; pp. 389-421.

123. Friesen, T.M. Resource structure, scalar stress, and the development of Inuit social organization. World Archaeol. 1999, 31, 21-37.

124. Parkinson, W.A. Tribal boundaries: Stylistic variability and social boundary maintenance during the transition to the Copper Age on the Great Hungarian Plain. J. Anthropol. Archaeol. 2006, 25, 33-58.

125. Ember, M. The relationship between economic and political development in nonindustrialized societies. Econ. Polit. Dev. 1963, 2, 228-248.

126. Feinman, G.M. Size, omplexity, and organizational variation: A comparative approach. Cross-Cultural Res. 2011, 45, 37-58.

127. Hoover, K.D. Microfoundational Programs. In Microfoundations Reconsidered: The Relationship of Micro and Macroeconomics in Historical Perspective; Duarte, P.G., Lima, G.T., Eds.; Edward Elgar Publishing Limited: Northampton, MA, USA, 2012; pp. 19-61. 
128. Janssen, M. Microfoundations: A Critical Inquiry; Routledge: London, UK, 1993.

129. Foster, J. Why is economics not a complex systems science? J. Econ. Issues 2006, 40, 1069-1092.

130. Foxon, T.J.; Kohler, J.; Michie, J.; Oughton, C. Towards a new complexity economics for sustainability. Cambridge J. Econ. 2013, 37, 187-208.

131. Krugman, P. The Self-Organizing Economy; Wiley-Blackwell: Oxford, UK, 1996; pp. 1-132.

132. Arthur, W.B. Competing technologies, increasing returns, and lock-in by historical events. Econ. J. 1989, 99, 116-131.

133. Schumpeter, J.A. The Theory of Economic Development; Harvard University Press: Cambridge, MA, USA, 1934.

134. Schelling, T.C. Micromotives and Macrobehavior; W.W. Norton: New York, NY, USA, 1978.

135. Arthur, W.B. Complexity and the economy. Science. 1999, 284, 107-109.

136. Loreau, M. Stability and Complexity of Ecoystems. In From Populations to Ecosystems; Princeton University Press: Princeton, NJ, USA, 2010; pp. 123-163.

137. Page, S.E. Diversity and Complexity (Primers in Complex Systems); Princeton University Press: Princeton, NJ, USA, 2010; pp. 1-296.

138. Lee, Y.; Amaral, L.A.N.; Canning, D.; Meyer, M.; Stanley, H.E. Universal features in the growth dynamics of complex organizations. Phys. Rev. Lett. 1998, 81, 3275-3279.

139. Di Guilmi, C.; Gaffeo, E.; Gallegati, M. Power law scaling in the world income distribution. Econ. Bull. 2003, 15, 1-7.

140. Hidalgo, C.A.; Hausmann, R. The building blocks of economic complexity. Proc. Natl. Acad. Sci. 2009, 106, 10570-10575.

141. Ormerod, P. Risk, recessions and the resilience of the capitalist economies. Risk Manag. 2010, 12, 83-99.

142. Karunanithi, A.T.; Garmestani, A.S.; Eason, T.; Cabezas, H. The characterization of socio-political instability, development and sustainability with Fisher information. Glob. Environ. Chang. 2011, $21,77-84$.

143. Peterson, G.D. Contagious disturbance, ecological memory, and the emergence of landscape pattern. Ecosystems 2002, 5, 329-338.

144. Biggs, D.; Biggs, R.O.; Dakos, V.; Scholes, R.J.; Schoon, M. Are we entering an era of concatenated global crises? Ecol. Soc. 2011, 16, 27.

145. Dunne, J.A.; Williams, R.J.; Martinez, N.D. Network structure and biodiversity loss in food webs: robustness increases with connectance. Ecol. Lett. 2002, 5, 558-567.

146. Hidalgo, C.A.; Klinger, B.; Barabási, A.-L.; Hausmann, R. The product space conditions the development of nations. Science. 2007, 317, 482-487.

147. Allen, C.R.; Angeler, D.G.; Garmestani, A.S.; Gunderson, L.H.; Holling, C.S. Panarchy: Theory and application. Ecosystems 2014, 17, 578-589.

(C) 2014 by the authors; licensee MDPI, Basel, Switzerland. This article is an open access article distributed under the terms and conditions of the Creative Commons Attribution license (http://creativecommons.org/licenses/by/4.0/). 